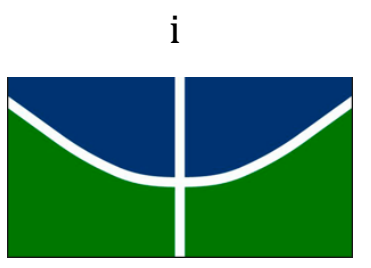

UNIVERSIDADE DE BRASÍLIA

PROGRAMA DE PÓS-GRADUAC̃̃O EM CIÊNCIAS MÉDICAS

FACULDADE DE CIÊNCIAS MÉDICAS

\title{
ANGIOPLASTIA COM IMPLANTE DE STENT EM PACIENTES COM ESTENOSE DE CARÓTIDA CERVICAL EM UMA SÉRIE MONOCÊNTRICA
}

\author{
FABIO SIMÕES FERNANDES \\ ORIENTADORA: IRUENA MORAES KESSLER \\ DISSERTAÇ̃̃O DE MESTRADO EM CIÊNCIAS MÉDICAS
}

Brasília-DF, 22 de Novembro - 2016 


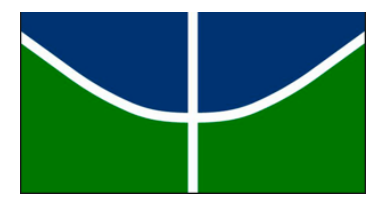

UNIVERSIDADE DE BRASÍLIA

PROGRAMA DE PÓS-GRADUAĈ̃̃ EM CIÊNCIAS MÉDICAS

FACULDADE DE CIÊNCIAS MÉDICAS

\author{
FABIO SIMÕES FERNANDES
}

\title{
ANGIOPLASTIA COM IMPLANTE DE STENT EM PACIENTES COM ESTENOSE DE CARÓTIDA CERVICAL EM UMA SÉRIE MONOCÊNTRICA
}

\begin{abstract}
Dissertação apresentada como requisito para a obtenção do Título de Mestre em Ciências Médicas pelo Programa de PósGraduação em Ciências Médicas da Universidade de Brasília.
\end{abstract}

Orientadora: Iruena Moraes Kessler 


\title{
ANGIOPLASTIA COM IMPLANTE DE STENT EM PACIENTES COM ESTENOSE DE CARÓTIDA CERVICAL EM UMA SÉRIE MONOCÊNTRICA
}

\author{
Dissertação apresentada como requisito \\ para a obtenção do Título de Mestre em \\ Ciências Médicas pelo Programa de Pós- \\ Graduação em Ciências Médicas da \\ Universidade de Brasília.
}

Defesa em 22 de novembro de 2016.

\section{BANCA EXAMINADORA}

\author{
Prof a . Dr. Iruena Moraes Kessler, presidente \\ Universidade de Brasília
}

Prof. Dr. Benício Oton de Lima

Universidade de Brasília

Prof. Dra. Adenalva Lima de Souza Beck

Universidade de Brasília 
À minha família, motivação principal para seguir em frente. À minha amada esposa Aline e à meus filhos Felipe e Rafael, sem os quais nada faria sentido. Aos meus pais, Armindo e Conceição que me tornaram o que sou. À minha irmã Andrea pelo eterno companheirismo. À Dra. Iruena, minha orientadora, professora, incentivadora e amiga. 


\section{RESUMO}

Introdução: O Acidente Vascular Encefálico (AVE) é a principal causa de doença incapacitante no Brasil. A Estenose Carotídea é importante causa de AVE isquêmico. Seu tratamento com Angioplastia de Carótida com Stent (ACS) é o tratamento menos invasivo, embora a eficácia de seus resultados ainda seja, em alguns artigos, inferiores a Endarterectomia. O presente estudo levantou uma série de casos de ACS em um hospital do Distrito Federal - DF, Brasil. Objetivo: Conhecer perfil demográfico e epidemiológico da população-alvo, identificando aspectos clínicos resultando em melhora na gestão de protocolos. Metodologia: 96 casos consecutivos submetidos a ACS entre janeiro de 2009 e dezembro de 2014. Desfecho primário: AVE isquêmico e morte. Desfecho secundário: complicações em sítio de punção, isquemia do miocárdio e outros eventos clínicos. Os dados clínicos, resultados e complicações foram avaliados durante a internação e após 30 dias. Resultados: 2,1\% (2 em 96) AVE's incapacitantes. Não houve óbito na amostra. Observou-se risco aumentado de complicações neurológicas nos pacientes acima de 80 anos $(p=0,015)$, assim como naqueles portadores de Insuficiência Renal Crônica ( $p=0,039)$. O Filtro de proteção cerebral (DPC) foi utilizado em $100 \%$ dos casos. Em $98,9 \%$ dos casos houve êxito no posicionamento do stent. Stents de malha fechada e aberta foram implantados em $89(92,7 \%)$ e 6 procedimentos $(6,3 \%)$, respectivamente. Conclusão: ACS em pacientes com CS se mostrou viável e segura, com baixos níveis de morbidade. A indicação de CS em pacientes acima de 80 anos e em portadores de Insuficiência Renal Crônica, deve ser criteriosa.

Palavras Chave: Acidente vascular cerebral, angioplastia de carótida, stent de carótida 


\section{ABSTRACT}

Introduction: Stroke is the main cause of incapacitating disease in Brazil. Carotid stenosis (CS) is an important cause of ischemic stroke. The treatment of CS with Carotid Stent Angioplasty (ACS) is the least invasive treatment, although the efficacy of its results is still, in some articles, inferior to Endarterectomy. The present study reported a series ACS cases in a hospital in the Federal District - DF, Brazil. Objective: To know the demographic and epidemiological profile of the target population, identifying clinical aspects resulting in improved protocol management. Methodology: 96 consecutive cases submitted to ACS between January 2009 and December 2014. Primary outcome: Ischemic stroke and death. Secondary outcome: complications at puncture site, myocardial ischemia and other clinical events. Clinical data, outcomes and complications were assessed during hospitalization and after 30 days. Results: $2.1 \%$ (2 out of 96 ) incapacitating stroke. There was no death in the sample. An increased risk of neurological complications in patients older than 80 years $(p=0.015)$ was observed, as well as in those with chronic renal insufficiency $(p=0.039)$. Cerebral protection filter (DPC) was used in $100 \%$ of the cases Stent placement was successful in $98.9 \%$ of the cases. Closed and open mesh stents were implanted in 89 (92.7\%) and 6 procedures $(6.3 \%)$, respectively. Conclusion: ACS in patients with CS was shown to be viable and safe, with low levels of morbidity. The indication of SC in patients older than 80 years and in patients with Chronic Renal Insufficiency should be judicious.

Keywords: Stroke ,Carotid stenting; Carotid stenosis; 


\section{LISTA DE FIGURAS E QUADROS}

Figura 1 - Dinâmica do fluxo na bifurcação carotídea. Local de menor fluxo e maior deposição das placas carotídeas. Retirado de: Tezduyar T.E., et al. 2007. 03

Figura 2. Técnica de endarterectomia. Lesão aterosclerótica envolvendo a Artéria Carótida Comum (CCA), Artéria Carótida Interna (ICA) e Artéria Carótida Externa (ECA) na primeira imagem. A imagem do meio é a representação da remoção da placa após incisão longitudinal da artéria. À direita, imagem do reparo da arteriotomia com o uso de enxerto (utilizado quando necessário) ' Retirado de: Roffi M., et al. 2009 .04

Figura 3. Procedimento de Angioplastia Carotídea com Implante de Stent. Após o cateterismo da Artéria Carótida Comum (CCA) com cateter guia, a lesão na Artéria Carótida Interna (ICA) é acessada e ultrapassada (A). Subsequente um stent autoexpansível é liberado geralmente revestindo a bifurcação (B e $C$ ). À seguir a dilatação por balão é realizada para atingir uma expansão do stent vencendo a resistência da placa de ateroma (D). ECA, Artéria Carótida Externa. Retirado de: Roffi M., et al. 2009. .05

Quadro 1- Distribuição no uso do Esquema de Antiagregação Plaquetária 15

Quadro 2. Distribuição das complicações relacionadas a ACS .16

Quadro 3. Distribuição de frequência dos cirurgiões. .17 


\section{LISTA DE TABELAS}

Tabela 1. Estatística descritiva da Idade dos pacientes por gênero

Tabela 2. Características dos pacientes segudo antecedentes patológicos por sexo

Tabela 3. Complicação neurológica por características da estenose carotídea.

Tabela 4. Características da doença ateromatosa carotídea por sexo 14

Tabela 5. Características dos Stents por sexo 14

Tabela 6. Estatística descritiva do tempo de internação e de procedimento........15

Tabela 7. Complicação neurológica por faixa etária 18

Tabela 8. Complicação neurológica por características da estenose carotídea..18

Tabela 9. Complicação neurológica por co-morbidades 19

Tabela 10. Distribuição do esquema antiagregação pré operatória por déficit neurológico pós operatório.

Tabela 11. Complicações pós operatórias durante a internação por comorbidades 20

Tabela 12. Complicações intraoperatórias por co-morbidades. 20 


\section{LISTA DE NOMENCLATURAS E ABREVIAÇÕES}

$\underline{\mathrm{AAS}}$ - Ácido Acetil Salicílico

$\underline{\text { ACS }}$ - Angioplastia de Carótida com Implante de Stent

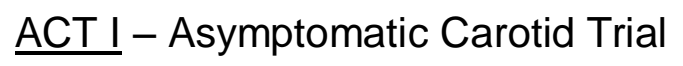

$\underline{\text { AIT }}$ - Acidente Isquêmico Transitório

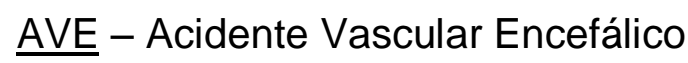

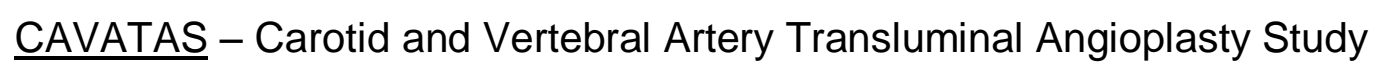

CCA - Artéria Carótida Comum

CREST - Carotid Revascularization Endarterectomy versus Stenting Trial

DATASUS - Sistema de Informação Institucional do Sistema Único de Saúde

DM - Diabetes Mellitus

DPC - Dispositivo de Proteção Cerebral

DPOC - Doença Pulmonar Obstrutiva Crônica

EC - Endarterectomia Carotídea

ECA - Artéria Carótida Externa

ERm - Escala de Rankin modificada

EVA-3S - Endarterectomy Versus Stenting in patients with Symptomatic Severe carotid Stenosis

$\underline{\text { HAS }}$ - Hipertensão Arterial Sistêmica

IC-DF - Instituto do Coração do Distrito Federal

ICA - Artéria Carótida Interna

$\underline{\text { ICSS }}$ - International Carotid Stenting Study

IM - Infarto do Miocárdio

$\underline{\mathrm{IRC}}$ - Insuficiência Renal Crônica

NASCET - North American Symptomatic Carotid Endarterectomy Trial

Protégé - Tipo de Stent Autoexpansível de Malha Aberta

SPACE - Stent-protected angioplasty versus carotid endarterectomy

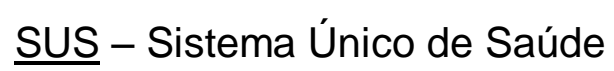

TCA - Teste de Coagulação Ativado

TOAST - Trial of Org in Acute Stroke Treatment.

WALLSTENT - Tipo de Stent Autoexpansível de Malha Fechada 


\section{SUMÁRIO}

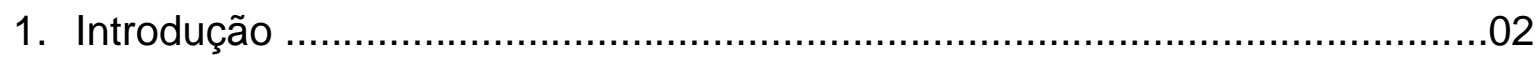

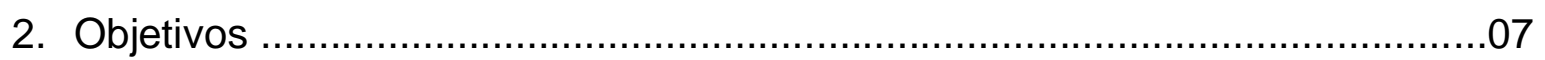

3. Pacientes e Métodos

3.1. Desenho

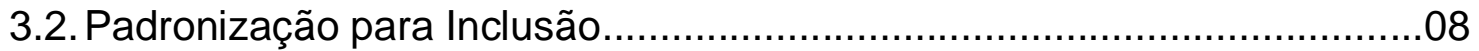

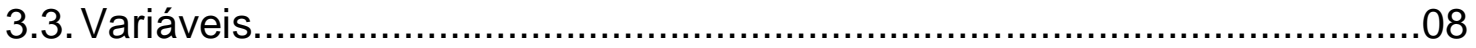

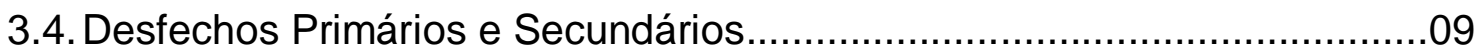

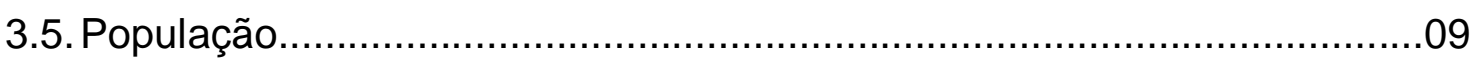

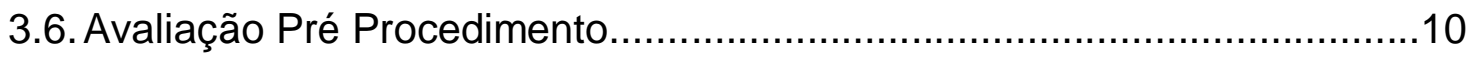

3.7. Protocolo Antiagregação Plaquetária.................................................10

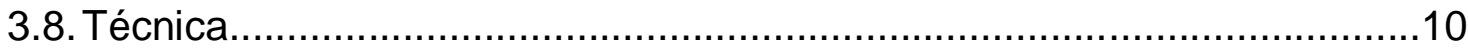

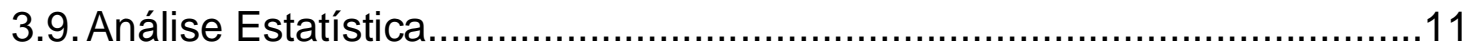

4. Resultados

4.1. Dados Demográficos

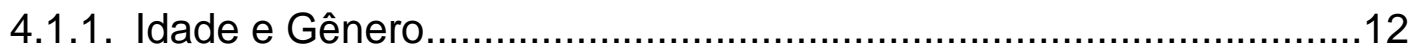

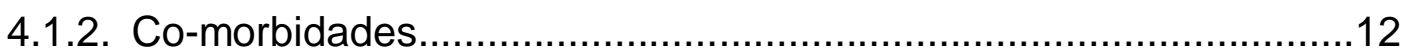

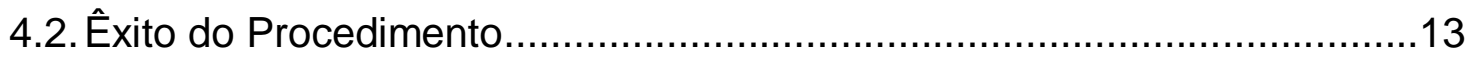

4.3. Tempo de Internação e Duração do Procedimento...................................14

4.4. Esquema de Antiagregação Plaquetária...............................................15

4.5. Complicações Não Neurológicas Intraoperatórias Perioperatórias..........16

4.6. Resultado Periprocedural de 30 dias..................................................17

4.7. Distribuição de Médicos Intervencionistas............................................17

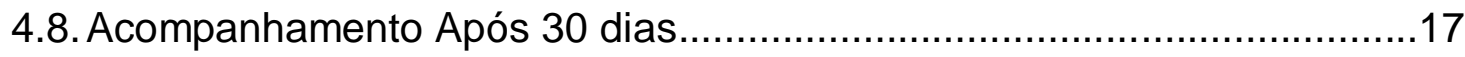

4.9. Correlações de Variáveis Associadas a Complicações

4.9.1. Correlação de Variáveis Associadas a Complicações

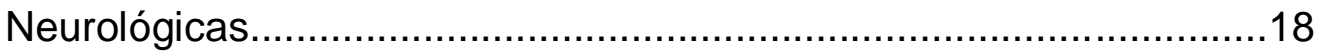

4.9.2. Correlação de Variáveis Associadas a Complicações Não

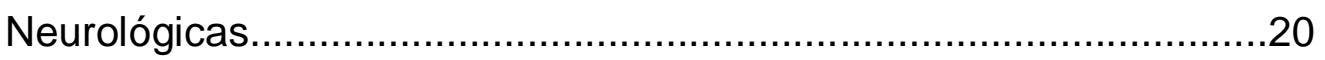

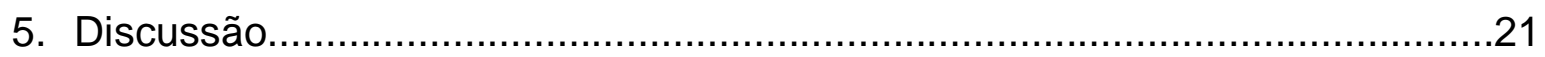

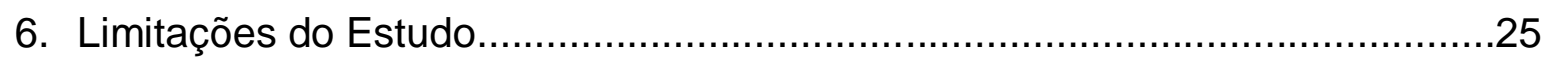

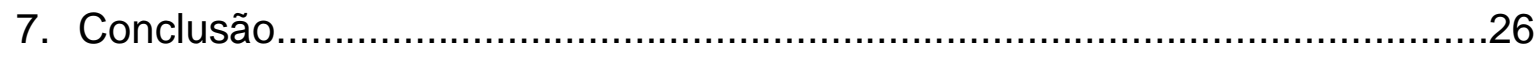

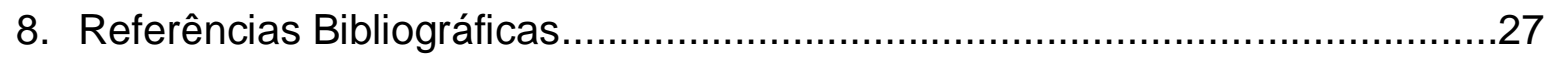




\section{1- INTRODUÇÃO}

O Acidente Vascular Encefálico (AVE) é, no Brasil, a principal causa de morte e incapacidade física adquirida no adulto, após os 40 anos. Segundo dados do DATASUS, são mais de $100 \mathrm{mil}$ mortes ao ano, correspondendo aproximadamente a $15 \%$ de todas as mortes ocorridas no país. Estima-se que $10 \%$ de todas as internações no SUS sejam por doença cerebrovasculares. Destes, uma grande parcela permanecerá com sequela neurológica permanente resultando em menos pessoas produtivas no mercado de trabalho e gerando custos de previdência precoce e reabilitação não mensurado pelas estatísticas locais (Lotufo 2005). O impacto desses custos nos Estados Unidos é estimada em 41 bilhões de dólares ao ano (Thom T. et al. 2006). A estenose carotídea aterosclerótica é uma das principais causas de eventos isquêmicos cerebrais, sendo a principal em idosos, segundo a Heart and Stroke Foundation of Canada - 2013.

A aterosclerose carotídea é um espessamento patológico da camada intimal das artérias carótidas comum ou interna, formando áreas focais conhecidas como placas (ou ateromas). Embora os ateromas possam permanecer estáveis durante vários anos, a ruptura superficial de placas instáveis (vulneráveis) leva à formação de trombos locais, com subsequente embolização para os territórios ipsilaterais das artérias carótida supraclinoidea, cerebral média ou cerebral anterior. Os sintomas resultantes são amaurose fugaz ipsilateral ou infarto da retina e ataque isquêmico transitório (AIT) ou AVE. O grau de estenose, mesmo que em placas estáveis, pode causar deficit neurológico, resultado de baixo fluxo sanguíneo, em geral em estenoses acima de 70\% (Fine-Eldestein J.S. - 1994).

Os fatores de risco mais relevantes, revisados por Mathiese E.B., em 2001, são idade, tabagismo, hipertensão arterial sistêmica (HAS), diabetes mellitus (DM), dislipidemia.

As placas carotídeas são frequentemente encontradas ao longo da parede interior da artéria carótida interna, oposta à bifurcação do fluxo, o que corresponde a uma área de baixa tensão de cisalhamento, de acordo com estudos de Tezduyar T.E. et al. 2007. (Figura 1) 


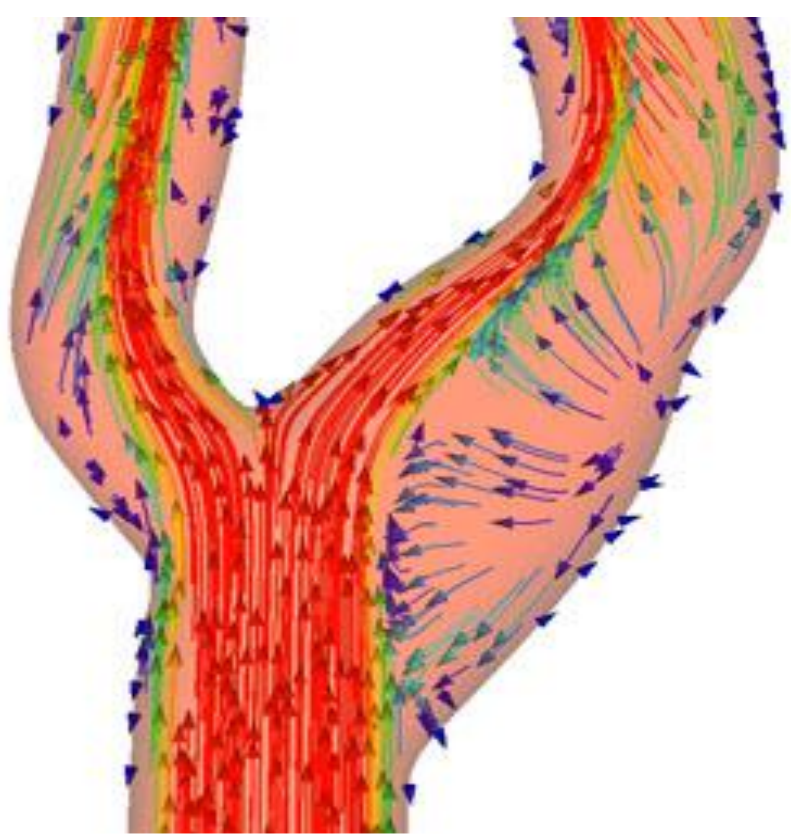

Figura 1 -Dinâmica do fluxo na bifurcação carotídea com o local de menor fluxo e maior deposição das placas carotídeas. Retirado de: Tezduyar T.E., et al. 2007

Alguns pacientes manifestam AIT ou AVE com mínima repercussão, cuja recuperação não imprime incapacidade significativa. Nesses pacientes, sabe-se que o risco de um novo evento isquêmico, com sequelas definitivas, é de $28 \%$ nos dois anos seguintes. O estudo TOAST - 1993, definiu a importância do tratamento incisivo nos pacientes de alto risco. Portanto, o tratamento da causa da isquemia tornou-se imperativo, tendo impacto superlativo na qualidade de vida e nos custos ao sistema de saúde.

O European Carotid Surgery Trial e o North American Symptomatic Carotid Endarterectomy Trial (NASCET), no início da década de 1990, demonstraram que o tratamento cirúrgico da estenose de carótida reduziu significativamente o risco de eventos isquêmicos encefálicos, quando comparado com a melhor terapia medicamentosa vigente. A terapia cirúrgica à época era a Endarterectomia Carotídea (EC), que consiste na abertura longitudinal da bifurcação carotídea e retirada mecânica da placa aterosclerótica com rafia primária (Figura 2). Houve portanto, após a EC, um risco estimado para AVE isquêmico ipsilateral de 2,8\% nos 3 anos seguintes ao procedimento, quando comparado com o risco de $16,8 \%$ ao ano dos pacientes submetidos a tratamento clínico. Assim, o tratamento cirúrgico das artérias carótidas se tornou padrão para os pacientes de alto risco. Esse tratamento oferece algum benefício em estenoses de $50 \%$ a $69 \%$ do lúmen 
da artéria carótida (em pacientes sintomáticos) e são altamente benéficos quando a estenose corresponde a $70 \%$ até $99 \%$, desde que não haja estenoses proximais significativas, de acordo com as diretrizes para prevenção de AVE em pacientes com isquemia ou AIT publicado na Stroke em 2006.
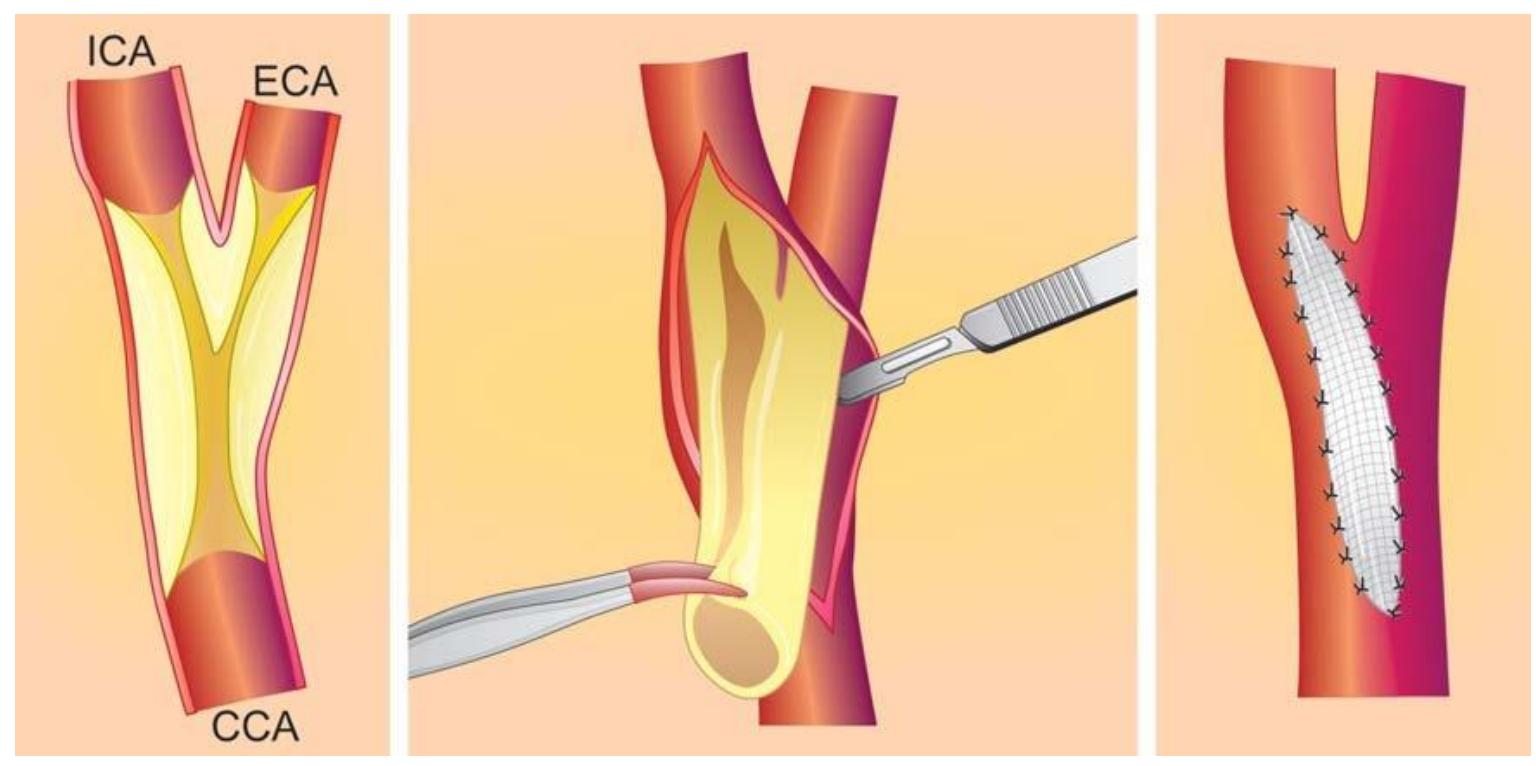

Figura 2. Técnica de endarterectomia. Lesão aterosclerótica envolvendo a Artéria Carótida Comum (CCA), Artéria Carótida Interna (ICA) e Artéria Carótida Externa (ECA) na primeira imagem. A imagem do meio é a representação da remoção da placa após incisão longitudinal da artéria. À direita, imagem do reparo da arteriotomia com o uso de enxerto (utilizado quando necessário)' Retirado de: Roffi M., et al. 2009

O sucesso da EC depende não somente do grau de estenose da carótida. Outros fatores, incluindo atraso na cirurgia após o evento inicial, gravidade do paciente, idade e co-morbidades associadas, limitam a indicação da cirurgia aberta, de acordo com revisão sistemática da Cochrane Database de 2011 sobre a EC na EC sintomática. Existe um significativo risco de AVE ou morte, resultado da cirurgia. Esse risco foi mensurado como $6 \%$ a $8 \%$ de morbidades maiores (AVE, Infarto do Miocárdio - IM) ou mortalidade. O risco de morbimortalidade (AVE isquêmico ipsilateral, IM e morte) nos primeiros 30 dias pós procedimento é em torno de 7,5\% (NASCET, 1991). Morbidades menores, incluindo paralisia de nervo craniano, hematoma de ferida e infecção da ferida operatória também fazem parte das possíveis morbidades pós operatórias (NASCET e European Carotid Surgery Trial de 1991). 
Neste cenário, com o desenvolvimento de técnicas endovasculares e a implementação de dispositivos mais seguros para o tratamento da ateromatose carotídea, a Angioplastia Carotídea com implante Stent (ACS) desponta como uma alternativa promissora à Endarterectomia em casos selecionados. A técnica minimamente invasiva consiste no posicionamento de stent endovascular ao nível da estenose e dilatação do mesmo com uso de balão, a fim de expansão da luz arterial (Figura 3).
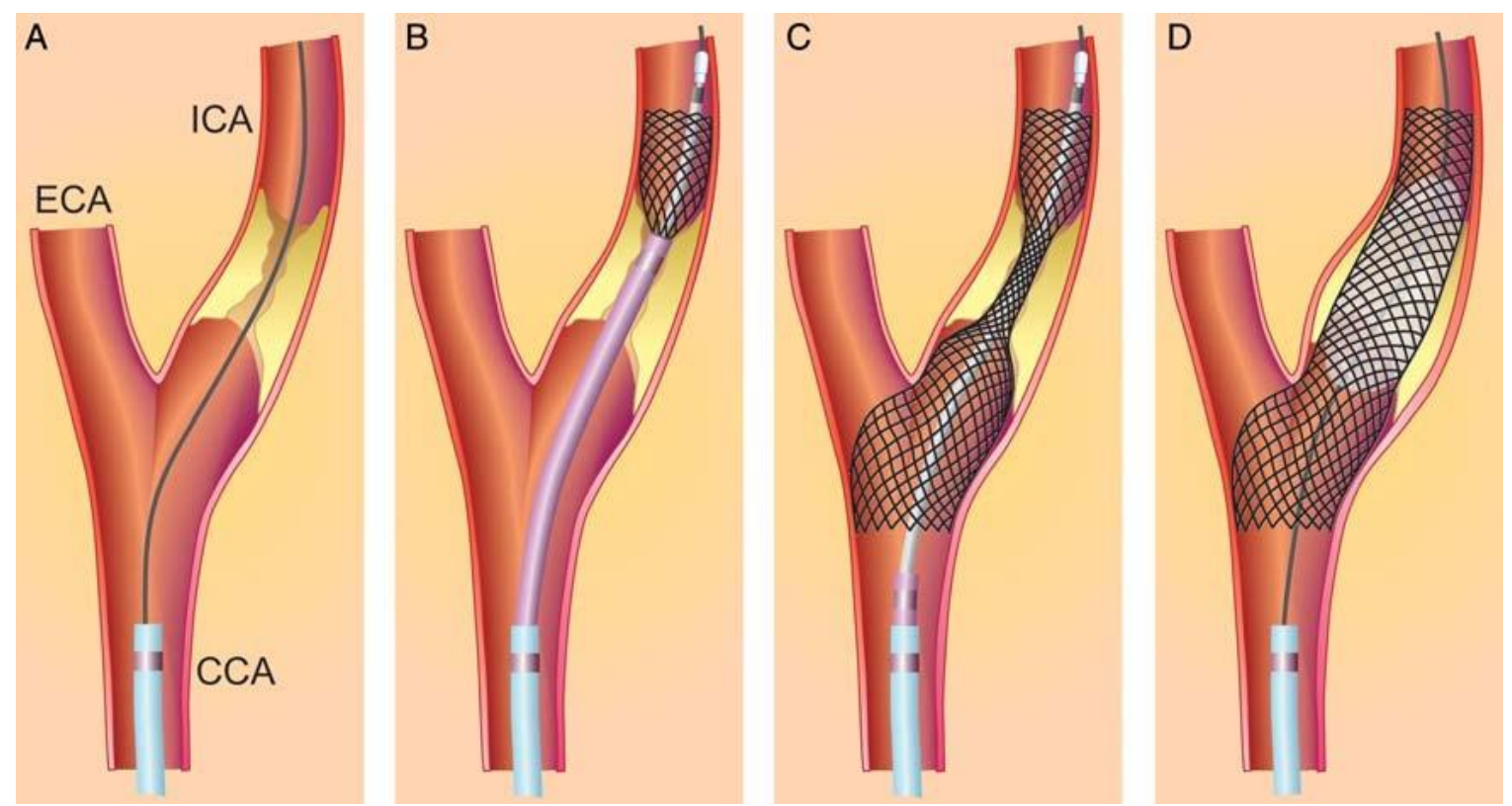

Figura 3. Procedimento de Angioplastia Carotídea com Implante de Stent. Após o cateterismo da Artéria Carótida Comum (CCA) com cateter guia, a lesão na Artéria Carótida Interna (ICA) é acessada e ultrapassada (A). Subsequente um stent auto-expansível é liberado geralmente revestindo a bifurcação (B e $C$ ). À seguir a dilatação por balão é realizada para atingir uma expansão do stent vencendo a resistência da placa de ateroma (D). ECA, Artéria Carótida Externa. Retirado de: Roffi M., et al. 2007.

Relatos iniciais que compararam as duas técnicas foram interrompidos por resultados muito discrepantes entre os grupos, com dados favoráveis à EC. O primeiro estudo concluído, de impacto na literatura, a explorar estes dados foi o Carotid and Vertebral Artery Transluminal Angioplasty Study (CAVATAS - 2009). Os autores demonstraram que o tratamento endovascular evitava de forma contundente as principais complicações inerentes à cirurgia aberta (lesão de nervos cranianos, IM, hematoma de leito cirúrgico e infecção), embora resultados de mortalidade e AVE em 30 dias tenha se mostrado inferior à técnica aberta. Nesse estudo, o tratamento endovascular com stent foi realizado em uma amostra 
minoritária e não equivalente ao grupo da EC. O grupo selecionado para tratamento endovascular foi dividido em dois subgrupos. O primeiro com o uso do balão isoladamente e o segundo com balão e stent. Na comparação entre os dois grupos, foi inequívoca a maior segurança no grupo com stent. Como consequência, a técnica com balão sem stent foi perdendo adeptos, sendo atualmente restrita a casos específicos. 


\section{2- OBJETIVOS}

O presente estudo tem como objetivos:

1- Conhecer perfil demográfico e epidemiológico da população submetida a ACS, identificando aspectos clínicos relevantes em um centro de referência de alta complexidade em tratamento de doenças cardiovasculares do Distrito Federal, Brasil.

2- Propor melhora na gestão de protocolos e formação de banco de dados com possibilidade de implementação nacional.

3- Identificar a taxa de morbimortalidade intraoperatória e pós operatória em até 30 dias pós procedimento e variáveis qualitativas e quantitativas relacionadas ao desfecho clínico da ACS incluindo: idade, gênero, comorbidades, déficits, escore na escala de Rankin modificada (ERm) na admissão e alta, estenose sintomática, esquema de antiagregação plaquetária, estenose contralateral, presença de placa ulcerada; complicações intraoperatórias; complicações pós operatórias imediatas e até 30 dias, tempo de internação, tipo de stent utilizado e uso de dispositivo de proteção cerebral (DPC). 


\section{3- PACIENTES E MÉTODOS}

\section{1- Desenho}

Trata-se de um estudo transversal, retrospectivo para avaliação do perfil demográfico e epidemiológico de pacientes submetidos à Angioplastia Percutânea de carótida com implante de Stent (ACS) no Instituto de Cardiologia do Distrito Federal (IC-DF), um instituto de alta complexidade e referência no tratamento de doenças cardiovasculares.

\section{2- Padronização para Inclusão}

Cada paciente assinou um termo de consentimento livre e esclarecido. A presente pesquisa foi aprovada pelo parecer com número CAAE IC-DF 57379716.1.0000.0026 do comitê de ética local do IC-DF. Todos os casos elegíveis foram selecionados por um grupo multidisciplinar, incluindo um neurologista e um neuroradiologista intervencionista. Todos os pacientes foram submetidos a exame por um neurologista antes e após o procedimento. O grau de comprometimento neurológico foi analisado utilizando a Escala de Rankin modificada (ERm) descrito por Swieten J.C. em 1988.

\section{3- Variáveis}

Foram analisadas variáveis qualitativas e quantitativas possivelmente associadas aos desfechos da ACS: idade, gênero, co-morbidades, déficits, escore na escala de Rankin modificada (ERm) na admissão e alta, estenose sintomática, presença de úlcera na placa aterosclerótica à angiografia, esquema de antiagregação plaquetária, estenose contralateral, complicações intra-operatórias; complicações pós operatórias imediatas e até 30 dias, tempo de internação, tipo de stent utilizado e uso de dispositivo de proteção cerebral (DPC). A escala de Rankin modificada encontra-se disponível no Anexo 01. 


\section{4- Desfechos Primários e Secundários}

Foi considerado como desfecho primário acidente vascular encefálico (AVE) isquêmico e/ou morte dentro de 30 dias após a ACS. O AVE foi definido como uma perturbação aguda da função neurológica focal como resultado de lesão vascular, com sintomas de duração superior à 24 horas. $O$ acidente vascular encefálico era considerado incapacitante se a pontuação na ERm fosse maior ou igual a três, pelo menos, 30 dias após o evento. Foram considerados desfechos secundários infarto do miocárdio (IM), ataque isquêmico transitório (AIT), lesão de pares cranianos, complicações locais relacionadas à punção e complicações sistêmicas dentro de 30 dias pós procedimento.

\section{5- População}

Todos os pacientes portadores de estenose da artéria carótida cervical sintomática ou assintomática que se apresentaram consecutivamente entre janeiro de 2009 e dezembro de 2014, submetidos à angioplastia de carótida cervical com implante de stent (ACS). Os dados foram coletados do prontuário eletrônico do referido hospital com o uso do programa de gestão hospitalar MV2000 (MV Sistemas - Porto Alegre, RS, Brasil).

Os pacientes foram considerados sintomáticos em caso de amaurose ipsilateral ou sintomas focais transitórios e/ou persistentes secundários à isquemia cerebral, até 180 dias prévios à intervenção. Os critérios de inclusão foram: estenose carotídea cervical $\geq 60 \%$ em pacientes com estenose sintomática ou $\geq$ $70 \%$ nas estenoses assintomáticas. Os pacientes considerados de maior risco cirúrgico à EC: reestenose após EC ou ACS, variantes anatômicas, estenose localizada na região submandibular ou adjacente à base do crânio, contraindicações para anestesia geral, estenose pós irradiação ou cirurgia, estenose associada proximal ou distal e oclusão total da artéria carótida contralateral. 


\section{6- Avaliação Pré Procedimento}

A lesão carotídea foi inicialmente diagnosticada por ultra-sonografia, angiotomografia ou angiorressonância e confirmada através de cateterismo seletivo das artérias carótidas comuns bilaterais e artérias vertebrais utilizando um angiógrafo (Integris, Allura, Philips Healthcare, Best, Holanda). O grau de estenose carotídea foi quantificado usando o critério do North America Symptomatic Carotid Endarterectomy Trial - NASCET, 1991.

\section{7- Protocolo de Antiagregação Plaquetária}

Todos os pacientes foram orientados a realizar o tratamento com doses diárias de 200mg de Ácido Acetil Salicílico e 75mg de Clopidogrel durante pelo menos 5 dias antes do procedimento, objetivando antiagregação plaquetária plena. Testes biológicos para avaliar a antiagregação plaquetária como o Teste de Coagulação Ativado (TCA) foram realizados à admissão. Em casos TCA < 250 segundos uma dose de ataque de $300 \mathrm{mg}$ de Clopidogrel e $200 \mathrm{mg}$ de Ácido Acetil Salicílico era administrada imediatamente antes do procedimento. Após o implante de stent, os pacientes continuaram a tomar $200 \mathrm{mg}$ ao dia de Ácido Acetil Salicilico indefinidamente e $75 \mathrm{mg}$ ao dia de Clopidogrel por pelo menos três meses. Durante a intervenção, um bolus intravenoso de 5000 UI de Heparina era administrada imediatamente após a colocação da bainha femoral.

\section{8- Técnica}

Os tratamentos foram realizados pela mesma equipe médica (IK, GP, GA e CS) em angiógrafo Integris, Philips Integris Allura (Healthcare, Best, Holanda) sob anestesia local ou, excepcionalmente, sob anestesia geral em pacientes não cooperativos ou com indicação de punção carotídea direta.

Após punção percutânea da artéria femoral, um introdutor 8F (Judkings, MN, USA) era posicionado e navegação de um catéter vertebral 5-F sob fio guia hidrofílico 0.035 de $260 \mathrm{~cm}$ (Terumo Corporation, Tóquio, Japão) para angiografia carotídea diagnóstica. Após o diagnóstico era posicionado um catéter guia Soft Tip 
7 ou $8 \mathrm{~F}$ (Stryker) na carótida comum ipsilateral ao lado a ser tratado. Usando uma técnica de road-mapping, a estenose era ultrapassada por um DPC de 3,5/5,5mm de diâmetro (Filter Wire EZ, Stryker, Natick, MA, EUA) e avançado até a carótida petrosa. Em casos de impossibilidade de avanço de filtro em estenoses severas, a mesma era atravessada por um fio guia 0,014 polegadas e realizado angioplastia com um balão de 30/20mm de diâmetro (gateway, Stryker, Natick, MA, EUA). Após o posicionamento do DPC um stent auto-expansível WALLSTENT (Stryker, Natick, MA, EUA) ou Protégé (Covidien, Playmouth, MN, EUA) era então avançado sobre o filtro e posicionado ao nível da estenose. A escolha do tipo de stent (aberto ou de célula fechada - vide Anexo 2) foi determinada de acordo com a disponibilidade no serviço e decisão do operador considerando a anatomia e morfologia da lesão. $O$ tamanho dos stents foi selecionado após a mensuração à angiografia. Após o implante do stent era realizada angioplastia com balão de $50 / 20 \mathrm{~mm}$ (gateway, Stryker, Natick, MA, EUA). A infusão endovenosa de $1 \mathrm{mg}$ de Atropina era administrada sistematicamente antes da angioplastia com balão em casos de estenose localizados no bulbo carótideo.

Ao término do procedimento, o dispositivo de hemostasia Angio-Seal (SaintJude Medical, MN, EUA) foi utilizado para a hemostasia femoral. Todos os pacientes foram internados em uma unidade de terapia intensiva durante pelo menos 24 a 48 horas após o tratamento endovascular. O seguimento clínico foi realizado após 30 dias, 6 meses e anualmente utilizando a ERm.

\section{9- Análise estatística}

Todos os dados foram analisados com base na intenção de tratar utilizando o Pacote de Software de Análise Estatística versão 22.0 IBM (SPSS, Chicago, IL, EUA). Foram realizados análise descritiva, teste t para comparação de médias de dois grupos, chi-quadrado exato de Fisher para avaliação de dependência entre variáveis. 


\section{4- RESULTADOS}

\subsection{Dados Demográficos}

\subsubsection{Gênero e Idade}

Um total de noventa e seis pacientes preencheram os critérios de inclusão. Houve predomínio masculino com 63 homens $(65,6 \%)$ e trinta e três mulheres (34,4\%), idade média de 69,8 \pm 9 ,8 anos (variação: $30-86$ anos). Não houve diferença estatística entre os gêneros com relação ao perfil etário - Tabela 1.

Tabela 1. Estatística descritiva da idade dos pacientes.

\begin{tabular}{lcccccc}
\hline Sexo & no & Média & Mediana & Mínimo & Máximo & p-valor* \\
\hline Total & 96 & 69,8 & 71,0 & 30 & 86 & - \\
Masculino & 63 & 69,4 & 71,0 & 30 & 86 & 0,623 \\
Feminino & 33 & 70,4 & 71,0 & 44 & 85 & \\
\hline
\end{tabular}

*p-valor do teste $t$ de comparação de médias

\subsubsection{Co-morbidades}

Dentre as co-morbidades mais frequentes predominou a Hipertensão Arterial Sistêmica com $79(82,3 \%)$ pacientes, a Dislipidemia $57(59,4 \%)$ e o Diabetes Mellitus com $33,3 \%$, ou 32 indivíduos. Essas e outras co-morbidades relevantes encontram-se na Tabela 2.

Tabela 2. Características dos pacientes segundo antecedentes patológicos por sexo.

\begin{tabular}{|c|c|c|c|c|c|c|c|}
\hline \multirow{2}{*}{ Variáveis } & \multicolumn{2}{|c|}{ Total } & \multicolumn{2}{|c|}{ Masculino } & \multicolumn{2}{|c|}{ Feminino } & \multirow{2}{*}{ p-valor* } \\
\hline & no & $\%$ & no & $\%$ & no & $\%$ & \\
\hline$\overline{\text { Total }}$ & 96 & 100,0 & 63 & 100,0 & 33 & 100,0 & \\
\hline Hipertensão arterial & 79 & 82,3 & 52 & 82,5 & 27 & 81,8 & 0,420 \\
\hline Diabetes melitus & 32 & 33,3 & 24 & 38,1 & 8 & 24,2 & 0,468 \\
\hline Displidemia & 57 & 59,4 & 37 & 58,7 & 20 & 60,6 & 0,805 \\
\hline Doença coronariana & 16 & 16,7 & 16 & 25,4 & 0 & 0,0 & 0,002 \\
\hline Fibrilação atrial & 4 & 4,2 & 3 & 4,8 & 1 & 3,0 & 1,000 \\
\hline Tabagismo & 31 & 32,3 & 23 & 36,5 & 8 & 24,2 & 0,342 \\
\hline Etilismo & 4 & 4,2 & 3 & 4,8 & 1 & 3,0 & 1,000 \\
\hline DPOC & 3 & 3,1 & 2 & 3,2 & 1 & 3,0 & 1,000 \\
\hline IRC & 6 & 6,3 & 4 & 6,3 & 2 & 6,1 & 1,000 \\
\hline
\end{tabular}

*p-valor do teste qui-quadrado

DPOC- Doença Pulmonar Obstrutiva Crônica; IRC: Insuficiência Renal Crônica 
Destes, 73 (76\%) eram assintomáticos e 23 (24\%) sintomáticos. Dentre os 23 pacientes sintomáticos, $13(56,4 \%)$ apresentaram acidente vascular cerebral e $10(43,6 \%)$ tiveram um AIT prévio à admissão. A maioria, 86 (89,6\%), apresentava bom estado clínico, ERm 1 e 2, durante o procedimento. Houve um predomínio de pacientes com estenose acima de $70 \%$, com 94 pacientes (97,8\%). Cinquenta e oito pacientes $(60,4 \%)$ tinham estenose carotídea (CS) no intervalo de $70 \%-89 \%$; $36(37,5 \%)$ tinham lesões pré- oclusivas, ou seja, maiores que $90 \%$ e $2(2,1 \%)$ entre 60-69\%. Dezoito indivíduos apresentavam lesão da artéria carótida contralateral com estenose acima de $50 \%$ e 9 desses $(9,4 \%)$ apresentando oclusão completa. Não houve correlação estatística destas variáveis com complicações neurológicas. Estes dados estão representados na Tabela 3.

Tabela 3. Complicação neurológica por características da estenose carotídea

\begin{tabular}{|c|c|c|c|c|c|c|c|}
\hline \multirow{2}{*}{ Variáveis } & \multicolumn{2}{|c|}{ Sim } & \multicolumn{2}{|c|}{ Não } & \multicolumn{2}{|c|}{ Total } & \multirow{2}{*}{ p-valor* } \\
\hline & no & $\%$ & n으 & $\%$ & $\mathrm{n}$ 응 & $\%$ & \\
\hline Total & 6 & 6,2 & 90 & 93,8 & 96 & 100,0 & - \\
\hline \multicolumn{8}{|c|}{ Estenose contra lateral (>50\%) } \\
\hline Sim & 2 & 33,3 & 26 & 28,9 & 28 & 29,2 & \\
\hline Não & 4 & 66,7 & 64 & 71,1 & 68 & 70,8 & 1,000 \\
\hline \multicolumn{8}{|c|}{ Estenose no lado do procedimento } \\
\hline Menor que : & 0 & 0,0 & 2 & 2,2 & 2 & 2,1 & \multirow{4}{*}{0,795} \\
\hline $70 \%$ a $79 \%$ & 1 & 16,7 & 27 & 30,0 & 28 & 29,2 & \\
\hline $80 \%$ a $89 \%$ & 3 & 50,0 & 29 & 32,2 & 32 & 33,3 & \\
\hline $90 \%$ e mais & 2 & 33,3 & 32 & 35,6 & 34 & 35,4 & \\
\hline \multicolumn{8}{|l|}{ Placa ulcerada } \\
\hline Sim & 2 & 33,3 & 20 & 22,2 & 22 & 22,9 & \multirow[b]{2}{*}{0,622} \\
\hline Não & 4 & 66,7 & 70 & 77,8 & 74 & 77,1 & \\
\hline \multicolumn{8}{|c|}{ Estenose sintomática } \\
\hline Sim & 2 & 28,6 & 21 & 23,3 & 23 & 24,0 & \multirow{2}{*}{0,632} \\
\hline Não & 5 & 71,4 & 68 & 75,6 & 73 & 76,0 & \\
\hline
\end{tabular}

\section{2- Êxito no procedimento:}

A taxa de sucesso técnico foi de $98,9 \%$, sendo implantados stents em 95 de 96 pacientes. Em 1 (1,1\%) paciente havia configuração anatômica desfavorável, com arco aórtico tipo III e angulação de mais de 60 graus entre a artéria carótida interna e a artéria carótida comum. Este paciente foi encaminhado para EC. Foram implantados stents de células abertas (Protégé, Covidien, Playmouth, MN, EUA) em $6(6,3 \%)$ casos e stents de célula fechada (Wallstent, Stryker, Natick, MA, EUA) em $89(92,7 \%)$ com utilização de CPDs em $100 \%$ dos procedimentos. Os DPC utilizados foram o Filter Wire (Stryker, Natick, MA, EUA) associado ao Wallstent e 
o Spider (Covidien, Playmouth, MN, EUA) ao Protégé. Dois (2,1\%) procedimentos precisaram de pré-dilatação antes da implantação do stent. As características relacionadas a placa ateromatosa (Tabela 4) e a material implantado (Tabela 5), não diferiram em distribuição na comparação de gêneros.

Tabela 4. Características da doença ateromatosa carotídea por sexo.

\begin{tabular}{|c|c|c|c|c|c|c|c|}
\hline \multirow{2}{*}{ Variáveis } & \multicolumn{2}{|c|}{ Total } & \multicolumn{2}{|c|}{ Masculino } & \multicolumn{2}{|c|}{ Feminino } & \multirow{2}{*}{ p-valor* } \\
\hline & no & $\%$ & $\mathrm{n}$ 응 & $\%$ & no & $\%$ & \\
\hline Total & 96 & 100,0 & 63 & 100,0 & 33 & 100,0 & - \\
\hline Sintomático & 23 & 24,0 & 12 & 19,0 & 11 & 33,3 & 0,208 \\
\hline Placa ulcerada & 22 & 22,9 & 13 & 20,6 & 9 & 27,3 & 0,515 \\
\hline Angioplastia contra-lateral prévia & 10 & 10,4 & 5 & 7,9 & 5 & 15,2 & 0,313 \\
\hline \multicolumn{8}{|l|}{ Porcentagem de estenose } \\
\hline Menor que $70 \%$ & 2 & 2,1 & 1 & 1,6 & 1 & 3,0 & \multirow{4}{*}{0,459} \\
\hline $70 \%$ a $79 \%$ & 27 & 28,1 & 19 & 30,2 & 8 & 24,2 & \\
\hline $80 \%$ a $89 \%$ & 31 & 32,3 & 22 & 34,9 & 9 & 27,3 & \\
\hline $90 \%$ e mais & 34 & 35,4 & 19 & 30,2 & 15 & 45,5 & \\
\hline \multicolumn{8}{|l|}{ Estenose contra-lateral } \\
\hline Menor ou igual a $50 \%$ & 68 & 70,8 & 44 & 69,8 & 24 & 72,7 & \multirow{3}{*}{0,819} \\
\hline Maior que $50 \%$ & 9 & 9,4 & 5 & 7,9 & 4 & 12,1 & \\
\hline Oclusão Total & 9 & 9,4 & 9 & 14,3 & 0 & 0,0 & \\
\hline
\end{tabular}

Tabela 5. Características dos Stents por sexo.

\begin{tabular}{|c|c|c|c|c|c|c|c|}
\hline \multirow{2}{*}{ Variáveis } & \multicolumn{2}{|c|}{ Total } & \multicolumn{2}{|c|}{ Masculino } & \multicolumn{2}{|c|}{ Feminino } & \multirow{2}{*}{ p-valor* } \\
\hline & $n$ ㅇ & $\%$ & $n$ ㅇ & $\%$ & $n \circ$ & $\%$ & \\
\hline Total** & 95 & 100,0 & 62 & 100,0 & 33 & 100,0 & - \\
\hline \multicolumn{8}{|c|}{ Tipo de stent } \\
\hline Protege & 6 & 6,3 & 4 & 6,5 & 2 & 6,1 & \\
\hline Walltent & 89 & 93,7 & 58 & 93,5 & 31 & 93,9 & 1,000 \\
\hline \multicolumn{8}{|c|}{ Tamanho do stent } \\
\hline $5 \times 40$ & 1 & 1,1 & 1 & 1,6 & 0 & 0,0 & \\
\hline $7 \times 30$ & 6 & 6,3 & 4 & 6,5 & 2 & 6,1 & \\
\hline $7 \times 40$ & 62 & 65,3 & 37 & 59,7 & 25 & 75,8 & \\
\hline $8 \times 40$ & 6 & 6,3 & 4 & 6,5 & 2 & 6,1 & 0,873 \\
\hline $9 \times 30$ & 1 & 1,1 & 1 & 1,6 & 0 & 0,0 & \\
\hline $9 \times 40$ & 18 & 18,9 & 13 & 21,0 & 4 & 12,1 & \\
\hline $9 \times 50$ & 1 & 1,1 & 1 & 1,6 & 0 & 0,0 & \\
\hline
\end{tabular}

\section{3- Tempo de Internação e Duração do Procedimento:}

Em 73 (76\%) pacientes o tempo de internação foi de aproximadamente $24 \mathrm{~h}$. Oitenta e dois pacientes, 85,4\% receberam alta dentro de $48 \mathrm{~h}$. Apenas 7 (7,3\%) pacientes necessitaram internação por mais de 3 dias. $O$ tempo médio de internação da amostra foi de 1,6 dias (desvio padrão de 1,6) - Tabela 6. Em 4 indivíduos não foi possível aferir tempo de internação, por serem pacientes internados em outras unidades. Esses foram transferidos para o IC-DF para 
realizar o procedimento, retornando ao hospital de origem após a recuperação pós anestésica.

O tempo médio do procedimento em 41(42,5\%) pacientes foi de 47,2 minutos (sd $\pm 21,6)$ variação de 25 a 152 minutos (Tabela 6). Não havia registro de tempo de procedimento antes de janeiro de 2014 (55 pacientes, ou 57,5\% da amostra).

Tabela 6. Estatística descritiva do tempo de internação e tempo de procedimento.

\begin{tabular}{lcccccc}
\hline Variáveis & no & Média & Mediana & $\begin{array}{c}\text { Desvio } \\
\text { Padrão }\end{array}$ & Mínimo & Máximo \\
\hline Tempo de procedimento & 41 & 47,2 & 43,0 & 21,6 & 25 & 152 \\
Tempo de internação & 92 & 1,6 & 1,0 & 1,6 & 1 & 10 \\
\hline
\end{tabular}

\section{4- Esquema de Antiagregação Plaquetária}

O uso do esquema correto foi comprovado em apenas 23 (24\%) dos pacientes, Enquanto o uso isolado do AAS ou do Clopidogrel foi de $24 \%$ e 5,2\% respectivamente. Em 6 casos $(6,2 \%)$ não havia dado sobre uso de medicação. A maioria dos pacientes (38 indivíduos - 39,6\%) não citavam nenhuma das duas medicações dentre as medicações de uso domiciliar. Um paciente $(1,0 \%)$ estava em uso de Dabigatrana (Quadro 1)

\begin{tabular}{|c|c|c|}
\hline Uso Antiagregante & no & $\%$ \\
\hline Total & 96 & $100 \%$ \\
\hline Sim & 52 & $54,2 \%$ \\
\hline AAS & 23 & $24,0 \%$ \\
\hline Clopidogrel & 5 & $5,2 \%$ \\
\hline AAS e Clopidogrel & 23 & $24,0 \%$ \\
\hline Outros & 1 & $1,0 \%$ \\
\hline Não & 38 & $39,6 \%$ \\
\hline Sem Informação & 6 & $6,2 \%$ \\
\hline
\end{tabular}

Quadro 1- Distribuição no uso do Esquema de Antiagregação Plaquetária AAS - Ácido Acetil Salićlico 


\section{5- Complicações Não Neurológicas Intraoperatórias e Perioperatórias:}

Trinta pacientes $(31,3 \%)$ apresentaram bradicardia no momento da abertura do stent revertido com Atropina $1 \mathrm{mg}$ endovenosa imediatamente, sem consequências hemodinâmicas ou neurológicas significativas. Em dois desses casos $(2,1 \%)$, houve hipotensão associada. Foi registrado um $(1,1 \%)$ caso de reação alérgica ao contraste, revertida prontamente. Um $(1,1 \%)$ caso de fratura do cateter guia ao nível femoral, com pronto resgate sem repercussão clínica e em um $(1,1 \%)$ caso o stent não foi posicionado devido anatomia desfavorável. (Quadro 2)

Dois $(2,1 \%)$ pacientes apresentaram hematoma no sítio de punção femoral. Um $(1,1 \%)$ paciente desenvolveu um pseudoaneurisma de artéria femoral que regrediu com compressão .

No pós operatório, cinco $(5,2 \%)$ pacientes apresentaram bradicardia e hipotensão, dois hipotensão isolada (um destes desenvolveu déficit neurológico no pós operatório). Um paciente cursou com hipertensão arterial necessitando de droga vasoativa. (Quadro 2)

\begin{tabular}{|lcc|}
\hline Complicações & $\mathrm{n}$ - & $\%$ \\
\hline Total & 96 & 100,0 \\
Complicações intraoperatórias & 1 & \\
Alergia & 28 & 1,0 \\
Bradicardia & 2 & 29,2 \\
Bradicardia e hipotensão & 1 & 2,1 \\
Corpo estranho & 2 & 1,0 \\
Déficit neurológico & 2 & 2,1 \\
Hipotensão & 60 & 2,1 \\
Não Bradicardia e Hipotensão & & 62,5 \\
Complicações pós-operatórias durante internação & 15 & 15,6 \\
Sim Hipotensão & 5 & 5,2 \\
$\quad$ Hipertensão & 2 & 2,1 \\
$\quad$ Sítio de Punção & 1 & 1,0 \\
Não Neurológico & 4 & 4,2 \\
Sem informação & 3 & 3,1 \\
Complicação neurológica em até 30 dias & 80 & 83,3 \\
Sim Não & 1 & 1,0 \\
\hline
\end{tabular}

Quadro 2. Distribuição das complicações relacionadas a CAS 


\section{6- Resultado Periprocedural de Trinta Dias}

A taxa de AVE global em 30 dias foi de 6,2\% (6/96 casos). Destes, em quatro houve piora neurológica persistente (piora no escore da ERm), mas apenas em dois houve sequela incapacitante (ERm:3). A mortalidade foi Zero na amostra. Não houve relato de IM.(Quadro 2)

\section{7- Distribuição de Médicos Intervencionistas}

Noventa e seis por cento (92/96) dos procedimentos foram realizados por apenas 4 profissionais, sendo que o profissional IK realizou 71,9\% (69/96) de todas as Angioplastias Carotídeas com Implante de Stent. A Distribuição entre os profissionais se encontra no Quadro 3.

\begin{tabular}{|lcc|}
\hline Cirurgião & no & \% \\
\hline Total & 96 & 100 \\
CS & 11 & 11,5 \\
GA & 3 & 3,1 \\
GP & 9 & 9,3 \\
IK & 69 & 71,9 \\
Outros & 4 & 4,2 \\
\hline
\end{tabular}

Quadro 3. Distribuição de frequência dos cirurgiões.

\section{8- Acompanhamento Após 30 dias}

Um total de $32(33,3 \%)$ pacientes foram acompanhados após o período periprocedural de 30 dias, com uma média de acompanhamento de tempo de 10,0 $\pm 2,2$ meses (intervalo: $1-21$ meses). Durante este tempo, um paciente $(1,04 \%)$ apresentou reestenose sintomática intra stent superior a $70 \%$ após 1 ano de ACS, evoluindo com AVE e deterioração no escore da ERm de 2 para 3. Necessitou de nova ACS. 


\section{9- Correlação de Variáveis Associadas a Complicações}

\subsection{1 - Correlacão de Variáveis Associadas a Complicacões Neurológicas}

A complicação neurológica e idade houve associação significativa. Dos seis casos de AVE, quatro tinham idade igual ou superior a 80 anos $(p=0,015)$ - Tabela 7. Não houve significância estatística relacionada ao tipo de stent, grau de estenose, grau de estenose contra-lateral, estenose sintomática ou placa ulcerada. (Tabela 8).

Tabela 7. Complicação neurológica por faixa etária.

\begin{tabular}{|c|c|c|c|c|c|c|c|}
\hline \multirow{2}{*}{ Variáveis } & \multicolumn{2}{|c|}{ Sim } & \multicolumn{2}{|c|}{ Não } & \multicolumn{2}{|c|}{ Total } & \multirow{2}{*}{ p-valor* } \\
\hline & no & $\%$ & no & $\%$ & no & $\%$ & \\
\hline Total & 6 & 6,3 & 88 & 91,7 & 96 & 100,0 & - \\
\hline \multicolumn{8}{|l|}{ Faixa etária } \\
\hline Menos de 60 anos & 0 & 0,0 & 11 & 100,0 & 11 & 100,0 & \multirow{4}{*}{0,015} \\
\hline 60 a 69 anos & 2 & 6,1 & 31 & 93,9 & 33 & 100,0 & \\
\hline 70 a 79 anos & 0 & 0,0 & 35 & 100,0 & 35 & 100,0 & \\
\hline 80 anos ou mais & 4 & 23,5 & 13 & 76,5 & 17 & 100,0 & \\
\hline
\end{tabular}

p-valor do teste qui-quadrado

Tabela 8. Complicação neurológica por características da estenose carotídea

\begin{tabular}{|c|c|c|c|c|c|c|c|}
\hline \multirow{2}{*}{ Variáveis } & \multicolumn{2}{|c|}{ Sim } & \multicolumn{2}{|c|}{ Não } & \multicolumn{2}{|c|}{ Total } & \multirow{2}{*}{ p-valor* } \\
\hline & no & $\%$ & no & $\%$ & no & $\%$ & \\
\hline Total & 6 & 6,2 & 90 & 93,8 & 96 & 100,0 & \\
\hline \multicolumn{8}{|c|}{ Estenose contra lateral (>50\%) } \\
\hline Sim & 2 & 33,3 & 26 & 28,9 & 28 & 29,2 & \\
\hline Não & 4 & 66,7 & 64 & 71,1 & 68 & 70,8 & 1,000 \\
\hline \multicolumn{8}{|c|}{ Estenose no lado do procedimento } \\
\hline Menor que : & 0 & 0,0 & 2 & 2,2 & 2 & 2,1 & \multirow{4}{*}{0,795} \\
\hline $70 \%$ a $79 \%$ & 1 & 16,7 & 27 & 30,0 & 28 & 29,2 & \\
\hline $80 \%$ a $89 \%$ & 3 & 50,0 & 29 & 32,2 & 32 & 33,3 & \\
\hline $90 \%$ e mais & 2 & 33,3 & 32 & 35,6 & 34 & 35,4 & \\
\hline \multicolumn{8}{|l|}{ Placa ulcerada } \\
\hline Sim & 2 & 33,3 & 20 & 22,2 & 22 & 22,9 & \multirow[b]{2}{*}{0,622} \\
\hline Não & 4 & 66,7 & 70 & 77,8 & 74 & 77,1 & \\
\hline \multicolumn{8}{|c|}{ Estenose sintomática } \\
\hline Sim & 2 & 28,6 & 21 & 23,3 & 23 & 24,0 & \multirow{2}{*}{0,632} \\
\hline Não & 5 & 71,4 & 68 & 75,6 & 73 & 76,0 & \\
\hline
\end{tabular}


Considerando as co-morbidades, um terço dos pacientes com Insuficiência renal crônica (IRC) apresentaram déficits neurológicos e complicações clínicas durante a internação e em até 30 dias do procedimento.

Quando comparamos exclusivamente complicações neurológicas e complicações pós operatórias observamos que IRC é fator de risco isolado $(p=0,039)$ - Tabela 9

Tabela 9. Complicação neurológica por comorbidades.

\begin{tabular}{lccccc}
\hline \multirow{2}{*}{ Comorbidades } & \multicolumn{3}{c}{ Sim } & \multicolumn{3}{c}{ Não } & p-valor* \\
\cline { 2 - 5 } & no & \% & no & \% & \\
\hline Hipertensão & 6 & 7,6 & 73 & 92,4 & 1,000 \\
Diabetes & 5 & 15,6 & 27 & 84,4 & 0,060 \\
Dislipidemia & 5 & 8,8 & 52 & 91,2 & 0,661 \\
Doença coronária & 1 & 6,3 & 15 & 93,8 & 1,000 \\
Fibilação & 1 & 25,0 & 3 & 75,0 & 0,221 \\
DPOC & 0 & 0,0 & 3 & 100,0 & 1,000 \\
IRC & 2 & 33,3 & 4 & 66,7 & 0,039 \\
\hline
\end{tabular}

Não obtivemos significância estatística na correlação entre déficit neurológico e o uso de antiagregante plaquetário. Embora o número de pacientes que necessitaram de dose de ataque imediatamente antes do procedimento tenha sido alta (Tabela 10).

Tabela 10. Distribuição do esquema antiagregação pré operatória por déficit pós operatório.

\begin{tabular}{|c|c|c|c|c|c|c|}
\hline \multirow{3}{*}{ Esquema } & \multirow{3}{*}{$\begin{array}{c}\text { Total } \\
\text { no }\end{array}$} & \multicolumn{4}{|c|}{ Déficit pós operatório } & \multirow{3}{*}{ p-valor* } \\
\hline & & \multicolumn{2}{|c|}{ Sim } & \multicolumn{2}{|c|}{ Não } & \\
\hline & & n으 & $\%$ & no & $\%$ & \\
\hline AAS & 23 & 1 & 4,3 & 22 & 95,7 & 0,424 \\
\hline Clopidogrel & 5 & 0 & 0,0 & 5 & 100,0 & 1,000 \\
\hline AAS+Clopidogrel & 23 & 1 & 4,3 & 22 & 95,7 & 0,424 \\
\hline Nenhum & 39 & 4 & 10,3 & 35 & 89,7 & 0,229 \\
\hline Sem informação & 6 & 0 & 0,0 & 6 & 100,0 & 1,000 \\
\hline
\end{tabular}

*p-valor do teste qui-quadrado exato de Fisher 
4.9.2 - Correlação de Variáveis Associadas a Complicações Não Neurológicas

Também observamos correlação entre IRC e complicações pós operatórias $(p=0,045)$. Não houve correlação significativa com relação às demais comorbidades (Tabela 11), exceto quando isolamos as complicações do intraoperatório. Neste grupo a Doença Coronariana foi predisponente $(p=0,047)$. Tabela 12

Tabela 11. Complicações pós-operatórias durante a internação por comorbidades.

\begin{tabular}{|c|c|c|c|c|c|}
\hline \multirow{2}{*}{ Comorbidades } & \multicolumn{2}{|c|}{ Sim } & \multicolumn{2}{|c|}{ Não } & \multirow{2}{*}{ p-valor* } \\
\hline & $\mathrm{n}$ 은 & $\%$ & no & $\%$ & \\
\hline Hipertensão & 15 & 19,0 & 64 & 81,0 & 0,589 \\
\hline Diabetes & 9 & 28,1 & 23 & 71,9 & 0,062 \\
\hline Dislipidemia & 9 & 15,8 & 48 & 84,2 & 0,752 \\
\hline Doença coronária & 2 & 12,5 & 14 & 87,5 & 1,000 \\
\hline Fibilação Atrial & 0 & 0,0 & 4 & 100,0 & 1,000 \\
\hline DPOC & 0 & 0,0 & 3 & 100,0 & 1,000 \\
\hline IRC & 3 & 50,0 & 3 & 50,0 & 0,045 \\
\hline Cirrose & 1 & 100,0 & 0 & 0,0 & 0,155 \\
\hline
\end{tabular}

*p-valor do teste qui-quadrado

DPOC- Doença Pulmonar Obstrutiva Crônica; IRC: Insuficiência Renal Crônica

Tabela 12. Complicações intraoperatórias por comorbidades.

\begin{tabular}{|c|c|c|c|c|c|}
\hline \multirow{2}{*}{ Comorbidades } & \multicolumn{2}{|c|}{ Sim } & \multicolumn{2}{|c|}{ Não } & \multirow{2}{*}{ p-valor* } \\
\hline & no & $\%$ & $n$ 은 & $\%$ & \\
\hline Hipertensão & 30 & 39,0 & 47 & 61,0 & 1,000 \\
\hline Diabetes & 14 & 45,2 & 17 & 54,8 & 0,489 \\
\hline Dislipidemia & 22 & 39,3 & 34 & 60,7 & 1,000 \\
\hline Doença coronária & 10 & 62,5 & 6 & 37,5 & 0,047 \\
\hline Fibilação Atrial & 2 & 50,0 & 2 & 50,0 & 0,644 \\
\hline DPOC & 1 & 33,3 & 2 & 66,7 & 1,000 \\
\hline IRC & 2 & 33,3 & 4 & 66,7 & 1,000 \\
\hline Cirrose & 1 & 100,0 & 0 & 0,0 & 0,393 \\
\hline
\end{tabular}

*p-valor do teste qui-quadrado

DPOC- Doença Pulmonar Obstrutiva Crônica; IRC: Insuficiência Renal Crônica 


\section{5- DISCUSSÃO}

A técnica de ACS, representa uma alternativa minimamente invasiva e menos traumática à cirurgia convencional para o reparo cirúrgico da estenose de carótida em casos selecionados. Esta técnica surgiu como uma alternativa de revascularização à EC em casos de estenose grave da artéria carótida interna em doentes com alto risco para a cirurgia aberta. Particularmente naqueles pacientes com co-morbidades significativas ou região cervical inapropriada devido a procedimentos cirúrgicos prévios ou irradiação, como discutido por Yadav em 2004 e Brott em 2010. Ambos procedimentos têm sido indicados pela certeza de agregar riscos menores que o tratamento clínico da estenose significativa da carótida, fato demonstrado por Rothwell na revista Lancet 2003. Por essa razão, ACS tem se tornado uma importante alternativa à endarterectomia a despeito das descritas complicações perioperatórias em séries não multicêntricas, por meio de metanálises como as de Luebke em.2007 e Bangalore em 2011.

A revisão sistemática da literatura sugere que o risco de acidente vascular cerebral ou morte após ACS é maior em pacientes com estenose sintomática, nos idosos e nos doentes com hipertensão ou história de doença arterial coronariana. Contrariamente à EC, (Roffi, 2009) o risco não está relacionado ao gênero ou à presença de oclusão da carótida contralateral. Neste estudo, a idade foi importante fator de risco para complicação neurológica $(p=0,015)$, juntamente com a presença de IRC $(p=0,039)$.

Estudos como endarterectomia versus angioplastia em pacientes com estenose grave e sintomática estenose de carótida (EVA-3S - 2006) e angioplastia percutânea com implante de stent da artéria carótida versus endarterectomia (SPACE - 2009) reportaram taxas de morte e acidente vascular encefálico com diferença significativa periprocedimento entre as duas técnicas $(9,6 \%$ na ACS e $7,6 \%$ na EC), nos 30 dias pós operatórios. Baseado nesses dados, Beckett et al. chamaram a atenção da comunidade científica para verificação da validade do tratamento endovascular em relação à Endarterectomia.

Alguns fatores, incluindo critérios de elegibilidade (Bosiers, 2010), anatomia desfavorável (Doig, 2015), uso da terapia antiagregante plaquetária, dilatação préoperatória (Stoeckel, 2004), tipo de stent escolhido (malha aberta ou fechada) e o uso de filtro de proteção cerebral (Tallarita 2011) influenciam no sucesso e 
evolução pós ACS. Assim, a evolução da técnica, a expertise dos profissionais e o desenvolvimento tecnológico dos dispositivos endovasculares resultou em mudança no perfil de resultados dos estudos ao longo das últimas duas décadas. Enquanto no início da primeira década os resultados mostravam-se desfavoráveis à ACS, atualmente existem estudos multicêntricos, randomizados e com poder estatístico que demonstram a equivalência entre as duas técnicas relacionados a resultados de curto (Kessler, 2013), médio (Ecker, 2007) e longo prazos (Timaran, do CREST 2013, Brott, do CREST 2016 e Rosenfield et al. do ACT I, 2016). Entretanto, ainda não é uniforme a consagração do ACS como técnica equivalente à EC (Feathestone do International Carotid Stenting Study - ICSS 2016).

No presente estudo, a uniformidade de materiais (92,7\% utilizado stent de malha fechada e $100 \%$ com DPC) e a pequena variabilidade de médicos intervencionistas ( $71,3 \%$ dos procedimentos realizados pelo mesmo profissional), podem ser pontuados como fatores que influenciam positivamente o resultado.

O perfil populacional de distribuição de gênero e idade, assim como o tempo de internação da nossa amostra foi congruente com a literatura atual. Devemos ressaltar que o perfil populacional que o IC-DF atende, difere da maioria dos serviços. Neste hospital, os paciente são geralmente referenciados para tratamento carotídeo, após investigação e tratamento de doenças cardíacas e/ou transplante cardíaco, hepático. Desta forma, inferimos que a população estudada tem um risco maior que a população em geral.

Os resultados da presente série apresentam taxa de mortalidade nula em 30 dias. Não houve piora neurológica avaliada pelo escore na escala de Rankin modificada, em 92 dos pacientes (95,8\%) em 30 dias, uma vez que dos 6 paciente com déficit no perioperatório, dois regrediram para a escala funcional pré procedimento. Apenas dois pacientes permaneceram com sequela incapacitante.

Com respeito a morbidades relacionadas ao sítio cirúrgico, encontramos $3,2 \%$ de complicações tratadas durante a internação. Estes dados são, via de regra, compatíveis com os estudos randomizados e multicêntricos mais recentes, cuja taxa de complicação relacionada ao ACS tem sido menor que a média histórica e estatisticamente similar à técnica aberta (EC).

Foi identificado que o paciente portador de IRC teve risco aumentado de eventos isquêmicos encefálicos e a complicações clínicas durante a internação $(p=0,039$ e $p=0,045$ respectimamente). A doença coronariana, aumentou o risco de 
complicação clínica intra-operatória $(p=0,047)$ relacionada ao manejo hemodinâmico (bradicardia, hipotensão), entretanto nessa amostra, sem repercussões no pós-operatório. Estes pacientes têm risco aumentado para quaisquer cirurgias, em especial procedimentos abertos. Qureshi em 2015 levanta alguns desses preditores na sobrevida em 5 anos. Mesmo cientes da gravidade deste grupo de pacientes, ainda não está definido na literatura se o tratamento clínico otimizado, no paciente com IRC, teria morbidade menor que o tratamento intervencionista na estenose carotídea. Novos estudos acerca deste tema seria de grande valia para a literatura médica.

Os pacientes com idade acima de 79 anos têm risco aumentado para eventos neurológicos periprocedimento para EC independente se EC ou ACS. Belkin em 2009 já questionava sobre a elevação da idade de risco de 60 para 80 anos. No presente estudo, os dados confirmaram risco aumentado nesta faixa etária pois de seis eventos neurológicos periprocedimento, quatro ocorreram em pacientes com 80 anos ou mais. $O$ aumento de eventos em octagenários se encontra bem estabelecido na literatura, sendo os artigos de Zahn em 2007 e Chiam em 2009 ilustrativos.

Nenhuma associação significativa foi encontrada entre o risco de acidente vascular cerebral ou morte, dentro de $24 \mathrm{~h}$ ou 30 dias, relacionada ao tipo de stent (malha fechada ou aberta), pré-dilatação ou utilização de um DPC. Todas as complicações neurológicas ocorreram em pacientes que receberam WALLSTENT com DPC. No entanto, esse material foi utilizado em $92,7 \%$ da casuística.

No International Carotid Stenting Study (ICSS), em 2016, houve impossibilidade de liberar o stent na região estenosada em $8 \%$ dos casos. $\mathrm{Na}$ nossa casuística há apenas 1 caso (1,1\%). Esta dado pode ser justificado por ser uma equipe única e experiente, com critérios estreitos de elegibilidade para 0 procedimento e o uso de DPC em $100 \%$ dos casos com predileção ao uso de stent de malha fechada.

Com relação a distribuição das co-morbidades, encontramos discrepâncias na incidência de pacientes com coronariopatias ou histórico de IM prévios. Este número na nossa amostra é três vezes menor comparado com estudos multicêntricos atuais (ACT I 2016, CREST 2016, ICSS 2016). A ausência de IAM na amostra, destoa novamente da literatura. Acreditamos que o viés cardiológico do hospital, onde o paciente é investigado e tratado previamente de complicações 
cardíacas antes de ser referenciado. Também ressaltamos os protocolos de prevenção de complicação cardiovasculares da equipe de Anestesiologia e Intensivismo, acompanhado no pós operatório por profissionais em equipe multiprofissional.

Por outro lado, a frequência nos eventos vasculares cerebrais relacionadas a ACS, $6,2 \%$ e complicações relacionadas a sítio de punção $(3,1 \%)$ vão ao encontro com a estatística apresentada nos últimos dois estudos randomizados multicêntricos (ACT 12016 e CREST 2016). Vale ressaltar que o índice de complicação com déficit incapacitante foi de 2,1\%, portanto com baixo impacto na população estudada. Os pacientes selecionados para ACS eram aqueles que não foram eleitos para EC de acordo com o descrito na metodologia. Inferimos portanto que os dados encontrados confirmam que a ACS é um método seguro mesmo nesta população.

Observamos que a dificuldade de realizar a dupla antiagregação no préoperatório, pode ter influenciado nos resultados de complicação neurológica. A dificuldade de realizar o planejamento medicamentoso pré e pós-operatório é multifatorial. Primeiro, a subnotificação no prontuário, dificulta a observação do real perfil de algumas variáveis, em especial aquelas que dependem da informação prestada pelo paciente ou familiares. Outro ponto é o perfil sócio-econômico da população alvo. Como o Clopidogrel é uma medicação que onera a maioria dos pacientes do IC-DF e não há dispensa deste fármaco nos programas de saúde pública do SUS, a maioria dos pacientes, ou não iniciam ou descontinuam seu uso. 


\section{6- LIMITAÇÕES DO ESTUDO}

Por se tratar de estudo retrospectivo, a falta de padronização na introdução dos dados no sistema leva a subnotificação ou notificação subjetiva em prontuário. Este foi um viés limitante neste estudo.

A perda de seguimento de uma parte significativa dos pacientes, impediu que fizéssemos a análise por tempo maior que 30 dias. 


\section{6- CONCLUSÃO}

Esse estudo descreveu um série monocêntrica de 96 pacientes submetidos à ACS em um hospital brasileiro. Houve um alto índice de sucesso técnico, com $98,9 \%$ de stents implantados e todos os pacientes utilizaram DPC. A taxa de AVE global em 30 dias foi de 6,2\% (6/96 casos), mas apenas com 2,1\% (2/96) de sequela incapacitante (ERm:3). A mortalidade foi inexistente na amostra no período avaliado. A idade acima de 80 anos e IRC foram variáveis associadas com maior fator de risco de complicações $(p=0,015)$ e $(p=0,039)$ respectivamente. Esse dados favorecem a ACS como um procedimento seguro e viável, porém com restrições a serem observadas em idosos acima de 80 anos e pacientes renais crônicos.

Os dados demográficos encontrados estão em consonância com aqueles reportados em grandes séries internacionais.

A padronização no registro dos dados, por meio da implementação de protocolos e questionários pré determinados pode resultar em melhora da notificação de informações importantes, resultando em banco de dados que orientem o perfil da população que melhor se beneficie da terapia proposta.

O uso de critérios de inclusão mais rígidos e a uniformização do uso da terapia antiagregante plaquetária na população do IC-DF, podem auxiliar na otimização dos resultados obtidos. Dessa forma, popusemos que haja políticas públicas para facilitar o acesso da população menos favorecida aos fármacos preconizados na literatura para a dupla antiagregação plaquetária. 


\section{8- REFERÊNCIAS BIBLIOGRÁFICAS}

Adams HP Jr, Bendixen BH, Kappelle LJ, Biller J, Love BB, Gordon DL, Marsh EE 3rd Classification of subtype of acute ischemic stroke. Definitions for use in a multicenter clinical trial. TOAST. Trial of Org 10172 in Acute Stroke Treatment. Stroke. 1993 Jan;24(1):35-41

Bangalore S., Kumar S., Wetterslev J., Bavry A.A., Gluud C., Cutlip D.E., Bhatt D.L., Carotid Artery Stenting vs Carotid Endarterectomy Meta-analysis and Diversity-Adjusted Trial Sequential Analysis of Randomized Trials; Arch Neurol,2011 Vol68, n02 172-84.

Beckett D, Gaines PA. Lessons from EVA-3S and SPACE. CardiovasC Intervent Radiol 2008;31:5-7

Belkin M., Bhatt D.L., Carotid Stenting in the Elderly Is 80 the New 60 ? Circulation. 2009;119:2302-2304.

Bonati LH, Ederle J, McCabe DJ, Dobson J, Featherstone RL, Gaines PA, Beard JD, Venables GS, Markus HS, Clifton A, Sandercock P, Brown MM; CAVATAS Investigators Long-term risk of carotid restenosis in patients randomly assigned to endovascular treatment or endarterectomy in the Carotid and Vertebral Artery Transluminal Angioplasty Study (CAVATAS): long-term follow-up of a randomised trial. Lancet Neurol. 2009 Oct;8(10):908-17.

Bosiers $M$, Deloose $K$, Peeters P. Carotid endovascular interventions: patient selection, devices, techniques and tips. Minerva Cardioangiol 2010;58:97-111.

Brott TG, Hobson 2nd RW, Howard G, Roubin GS, Clark WM, Brooks W, et al. Stenting versus endarterectomy for treatmentof carotid artery stenosis. $N$ Engl J Med 2010;363:11-23). 
Brott T.G., Howard G., Roublin G.S., Meschia J.F., Mackey A., Brooks W., Moore W.S., Hill M.D., Mantese V.A., Clark W.M., Timaran C.H., Heck D., Leimgruber P.P., Sheffet A.J., Howard V.J., Chaturvedi S., Lal B.K., Voeks J.H., Hobson II R.W. Long-Term Results of Stenting versus Endarterectomy for Carotid-Artery Stenosis for the CREST Investigators, $N$ Eng J Med 2016, 374;11

Chiam PT, Roubin GS, Panagopoulos G, lyer SS, Green RM, Brennan C, Vitek JJ. One-year clinical outcomes, midterm survival, and predictors of mortality after carotid stenting in elderly patients. Circulation 2009;28:2343-2348.

Doig D, Hobson B.M., Müller M., Jäger H.R., Featherstone R.L., Brown M.M., Bonati L.H., Richards T. on behalf of the ICSS-MRI Study Investigators, Carotid Anatomy Does Not Predict the Risk of New Ischaemic Brain Lesions on Diffusion-Weighted Imaging after Carotid Artery Stenting in the ICSS-MRI Substudy Eur J Vasc Endovasc Surg 2015 1-7

Ecker R., Lau T., Levy E.I., Hopkins N., Thirty-day morbidity and mortality rates for carotid artery intervention by surgeons who perform both carotid endarterectomy and carotid artery angioplasty and stent placement $J$ Neurosurg 2007, 106:217-221,

Eckstein HH, Ringleb P, Allenberg JR, Berger J, Fraedrich G, Hacke W, et al. Results of the Stent-protected angioplasty versus carotid endarterectomy (SPACE) study to treat symptomatic stenoses at 2 years: a multinational, prospective, randomized trial. Lancet Neurol 2009;8:135

European Craotid Surgery Trialists Collaborative Group. MRC European Carotid Surgery Trial: interim results for symptomathic pacientes with severe $(\mathbf{7 0}-99 \%)$ or with mild $(0-29 \%)$ carotid stenosis -Lancet $1991 ; 337$ : 1235-4 
Fine-Edelstein JS, Wolf PA, O'Leary DH, Poehlman H, Belanger AJ, Kase CS, et al. Precursors of extracranial carotid atherosclerosis in the Framingham Study. Neurology 1994;44:1046-50

Feathestone R.L., Dobson J., Ederle J, Doig D., Bonati L.H., Morris S., Patel N.V., Brown M.M., on behalf of the ICSS-MRI Study Investigators Carotid artery stenting compared with endarterectomy in patients with symptomatic carotid stenosis (International Carotid Stenting Study ICSS): a randomised controlled trial with cost-effectiveness analysis Health Technology Assessment NIHR Journals Library 2016, Vol. 20, n. 20

Heart and Stroke Foundation of Canada. Stroke statistics. 2010. http://www.heartandstroke.com (last accessed 17 May 2013).

Kessler I.M., Gory B., Macian F., Nakiri G., Al-Khawaldeh M., Riva R., Boncoeur M.P., Mounayer C., Carotid artery stenting in patients with symptomatic carotid stenosis: A single-center series Journal of Neuroradiology 2013, 40, 38-44

Lotufo P. Stroke: a neglected disease in Brazil. São Paulo Med J. 2005;123(1):3-4.

Luebke, ${ }^{*}$ M. Aleksic and J. Brunkwall ;Meta-analysis of Randomized Trials Comparing Carotid Endarterectomy and Endovascular Treatment T. Eur J Vasc Endovasc Surg 2007; 34, 470 - 479

Mas JL, Chatellier G, Beyssen B, Branchereau A, MoulinT, Becquemin JP, et al. Endarterectomy versus stenting in patients with symptomatic severe carotid stenosis - EVA-3S. N Engl J Med 2006;355:1660—71

Mathiesen EB, Joakimsen O, Bonaa KH Prevalence of and risk factors associated with carotid artery stenosis: the Tromso Study. Cerebrovasc Dis 2001;12:44-51 
Ministério da Saúde/SE/Datasus (endereço na internet).Local: Sistema de Informações Hospitalares do SUS - SIH/SUS IBGE: base demográfica. ww2.datasus.gov.br/DATASUS/index.php?area $=02$ )

North American Symptomatic Carotid Endarterectomy Trial (NASCET) Collaborators. Beneficial effect of carotid endarterectomy in symptomatic patients with high-grade carotid stenosis. N Engl J Med 1991;325:445—53.

Qureshi A.I., Chaudhry S.A., Qureshi M.H., Suri M.F.K., Rates and Predictors of 5-Year Survival in a National Cohort of Asymptomatic Elderly Patients Undergoing Carotid Revascularization Neurosurgery 2015, 76:34-41,

Rerkasem K, Rothwell PM. Carotid endarterectomy for symptomatic carotid stenosis. Cochrane Database Syst Rev. 2011;(4)

Roffi M., Mukherjee D., Clair D.G. Carotid artery stenting vs. endarterectomy European Heart Journal (2009) 30, 2693-2704

Rosenfield K., Matsumura J.S., Chaturvedi S., Riles T., Ansel G.M., Metzger C., Wechsler L., Jaff M.R., Gray W. Randomized Trial of Stent versus Surgery for Asymptomatic Carotid Stenosis for the ACT I Investigators, N Eng J Med 2016, 374;11

Rothwell PM, Eliasziw M, Gutnikov SA, Fox AJ, Taylor DW, Mayberg MR, et al. Analysis of pooled data from the randomised controlled trials of endarterectomy for symptomatic carotid stenosis. Lancet 2003;361:107-16.

Sacco RL, Adams R, Albers G, et al. Guidelines for prevention of stroke in patients with ischemic stroke or transient ischemic attack. Stroke. 2006;37:577-61.

Stoeckel D, Pelton A, Duerig T. Self-expanding nitinol stents: material and design considerations. Eur Radiol 2004;14:292

Tallarita T, Rabinstein AA, Cloft H, Kallmes D, Oderich GS, Brown RD, et al. Are distal protection devices 'protective' during carotid angioplasty and 
stenting? Stroke 2011;42:1962-6.

Tezduyar TE, Sathe S, Cragin T, Nanna B, Conklin BS, Pausewang J, Schwaab M, "Modeling of Fluid-Structure Interactions with the Space-Time Finite Elements: Arterial Fluid Mechanics", International Journal for Numerical Methods in Fluids, 54 (2007) 901-922.

Timaran C.H., Mantese V.A., Malas M., Brown O.W., Lal B.K., Moore W.S., Voeks J.H., Brott T.G., Differential Outcomes of Carotid Stenting and Endarterectomy Performed Exclusively by Vascular Surgeons in the Carotid Revascularization Endarterectomy versus Stenting Trial (CREST) for the CREST Investigators J. Vasc Surg. 2013 57(2): 303-308.

Thom T, Haase N, Rosamond W, et al. Heart disease and stroke statistics 2006 update: a report from the American Heart Association Statistics Committee and Stroke Statistics Subcommittee. Circulation. 2006;113:e85e151

van Swieten JC,Koudstall PJ,Visser MC,Schouten HJA,van Gijn J. Interobserver agreement for the assessment of handicap in stroke patients. Stroke 1988;19:604-607

Yadav JS, Wholey MH, Kuntz RE, Fayad P, Katzen BT, Mishkel GJ, et al. Protected carotid artery stenting versus endarterectomy in high-risk patients. N Engl J Med 2004;351:1493—501.

Zahn R, Ischinger T, Hochadel M, Zeymer U, Schmalz W, Treese N, Hauptmann KE, Seggewiss H, Janicke I, Haase H, Mudra H, Senges J. Carotid artery stenting in octogenarians: results from the ALKK Carotid Artery Stent (ACS) Registry. Eur Heart J 2007;28:370-375. 
ANEXO 1

\begin{tabular}{|c|l|}
\hline \multicolumn{2}{|c|}{ ESCALA DE RANKIM MODIFICADA } \\
\hline ESCORE & \multicolumn{1}{|c|}{ DESCRIÇÃO } \\
\hline 0 & Sem Sintomas \\
\hline 1 & $\begin{array}{l}\text { Sintomas, entretanto sem significativa limitação. } \\
\text { Capaz de exercer todas as atividades cotidianas e laborais }\end{array}$ \\
\hline 2 & $\begin{array}{l}\text { Limitação leve. } \\
\text { Incapaz de exercer todas as atividades cotidianas, mas capaz de } \\
\text { realizar atividades de auto-cuidado sem assistência }\end{array}$ \\
\hline 3 & $\begin{array}{l}\text { Limitação Moderada } \\
\text { Necessita de alguma ajuda, mas capaz de deambular sem } \\
\text { assistência }\end{array}$ \\
\hline 4 & $\begin{array}{l}\text { Limitação Moderadamente Grave } \\
\text { Incapaz de deambular sem assistência e incapaz de realizar auto- } \\
\text { higiene sem auxílio }\end{array}$ \\
\hline 5 & $\begin{array}{l}\text { Limitação Grave } \\
\text { Acamado, incontinente e requerendo cuidados de enfermagem } \\
\text { constantes }\end{array}$ \\
\hline 6 & Morte \\
\hline
\end{tabular}

van Swieten JC,Koudstall PJ,Visser MC,Schouten HJA,van Gijn J. Interobserver agreement for the assessment of handicap in stroke patients. Stroke 1988;19:604-607 


\section{ANEXO 2}

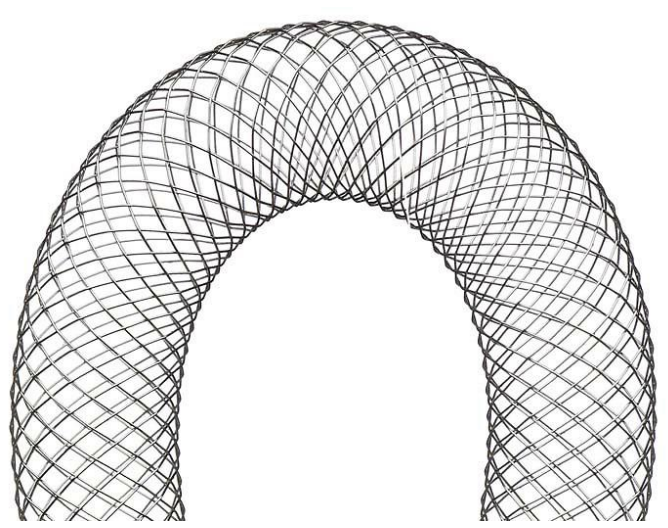

Wallstent - Boston Cientific ${ }^{(\circledast)}$

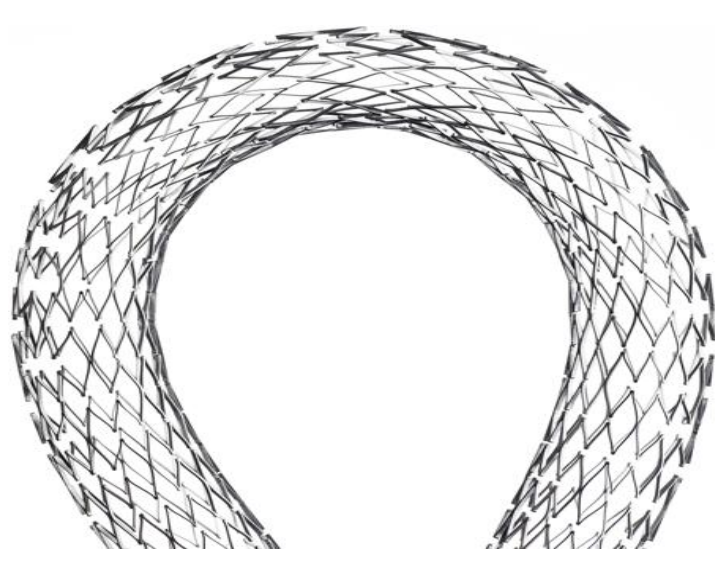

Protégé Stent - Covidien ${ }^{\circledR}$

Os stents de malha fechada, como Wallstent, têm menores taxas de complicações pós operatórias comparativamente com os de malha aberta, como o Protégé, tendo em vista que a fragmentação da placa aterosclerótica e consequente embolização de ramos distais é menor no primeiro exemplo.

Tallarita T, Rabinstein AA, Cloft H, Kallmes D, Oderich GS, Brown RD, et al. Are distal protection devices 'protective' during carotid angioplasty and stenting? Stroke $2011 ; 42: 1962-6$. 